\title{
Chemoradiotherapy in the Treatment of Nasopharyngeal Carcinoma
}

This article was published in the following Dove Press journal:

Cancer Management and Research

\author{
Zhong-Guo Liang ${ }^{1} *$ \\ Fan Zhang ${ }^{2, *}$ \\ Bin-Bin Yu ${ }^{1} *$ \\ Ling $\mathrm{Li}^{1}$ \\ Song $\mathrm{Qu}^{\prime}$ \\ Ye $\mathrm{Li}^{1}$ \\ Ying Guan' \\ Ren-Ba Liang' \\ Lu Han' \\ Xiao-Dong Zhu (iD) \\ 'Department of Radiation Oncology, \\ Guangxi Medical University Cancer \\ Hospital, Nanning, People's Republic of \\ China; ${ }^{2}$ Microbiome Research Centre, St \\ George and Sutherland Clinical School, \\ The University of New South Wales \\ Sydney, St George Hospital, Kogarah, \\ NSW, Australia \\ *These authors contributed equally to \\ this work
}

Correspondence: Xiao-Dong Zhu Department of Radiation Oncology, Guangxi Medical University Cancer Hospital, 7I He Di Road, Nanning 53002I, People's Republic of China Tel +86-77I-533I466

Email zhuxdonggxmu@I26.com
Purpose: To construct a prognostic index (PI) for overall survival (OS) to stratify nasopharyngeal carcinoma (NPC) into high-risk and low-risk groups. We also applied the model to investigate the role of the addition of adjuvant chemotherapy (AC) to concurrent chemoradiotherapy (CCRT) regimens for the treatment of NPC.

Methods: A prognostic model was established based on a retrospective study of 362 patients from January 2008 to June 2011. The discriminative and calibration abilities of the model were evaluated by Harrell's concordance index (C-index), and calibration curves. Bootstrapping was used to perform for internal validation. External validation was conducted using 324 patients diagnosed with NPC from July 2011 to December 2012 at the same institution. Survival analyses were performed between CCRT-AC and CCRT alone groups for the high-risk and low-risk groups.

Results: The primary PI comprised covariates that were associated with OS in the training cohort, including $\mathrm{T}$ stage, $\mathrm{N}$ stage, age, and plasma alkaline phosphatase (ALP). Internal and external validation showed that the discrimination of the PI for OS was significantly better than that of the 8 th edition AJCC staging system. Discretization by using a fixed PI score cut-off of 407.96 determined from the training data set yielded high- and low-risk subgroups with distinct OS outcomes in the validation cohort. Adjuvant chemotherapy improved OS in high-risk patients (HR $0.620,95 \%$ CI 0.408 to $0.941 ; \mathrm{P}=0.023$ ) but increased the risk of distant metastasis (HR, 4.222, 95\% CI, 0.959 to 18.585 ; $\mathrm{P}=0.038$ ) in low-risk patients.

Conclusion: The proposed prognostic model achieved good prediction and calibration of OS for patients with NPC. The addition of adjuvant chemotherapy might be a double-edged sword, bringing survival benefit to high-risk patients but greater risk of distant metastasis to low-risk patients.

Keywords: nasopharyngeal carcinoma, prognostic model, concurrent chemoradiotherapy, adjuvant chemotherapy

\section{Introduction}

Nasopharyngeal carcinoma (NPC) is prevalent in south-eastern China, Malaysia, Indonesia, Singapore, Eastern Asia, and Northern Africa. According to surveys from the International Agency for Research on Cancer, there are an estimated 129,079 new cases and 72,987 related deaths each year. ${ }^{1}$

Radiation therapy has been the mainstay of curative treatment for NPC for decades. Currently, intensity-modulated radiotherapy (IMRT) is widely utilized, 
which significantly improves coverage of the target tumour and spares normal structures, leading to increased localregional control and reduced radiotherapy-related toxicities. Moreover, the addition of chemotherapy has increased the survival time of NPC patients. $^{2-4}$

Clinically, patients with the same TNM stage may have different prognoses, which indicates that some other factors should be integrated to improve the prediction of patient outcomes. Recent studies have reported that several relevant variables are associated with patient survival, including pretreatment serum lactate dehydrogenase $(\mathrm{LDH}),{ }^{5}$ leucopaenia, ${ }^{6}$ anaemia, ${ }^{7}$ and alkaline phosphatase (ALP). ${ }^{8}$

In addition, a good model should not only help predict prognosis but also help guide clinicians in making treatment decisions. At present, there is controversy regarding the role of adjuvant chemotherapy (AC) after concurrent chemoradiotherapy (CCRT) for NPC. For example, a multicentre randomized controlled trial observed no significant survival benefits from the addition of adjuvant chemotherapy to CCRT. $^{9}$ A retrospective study with
2263 patients also compared CCRT plus adjuvant chemotherapy versus CCRT alone and found no survival benefit from adjuvant chemotherapy. ${ }^{10}$ In contrast, a multiinstitutional retrospective study with 380 patients in the CCRT alone arm and 327 patients in the CCRT-AC arm showed that AC can significantly improve survival. ${ }^{11}$

The purpose of this study was to define possible predictors of overall survival (OS) in NPC patients treated with IMRT and to establish an effective prognostic model to stratify high-risk and low-risk groups by OS, which might provide an individualized prediction for treatment outcomes. Furthermore, we aimed to apply the model to investigate the role of the addition of $\mathrm{AC}$ to CCRT regimens for the treatment of NPC.

\section{Methods}

\section{Patients}

Between January 2008 and December 2012, 686 patients who were newly diagnosed with non-metastatic NPC were retrospectively reviewed. Of these, 362 patients, treated from January 2008 to June 2011, comprised the

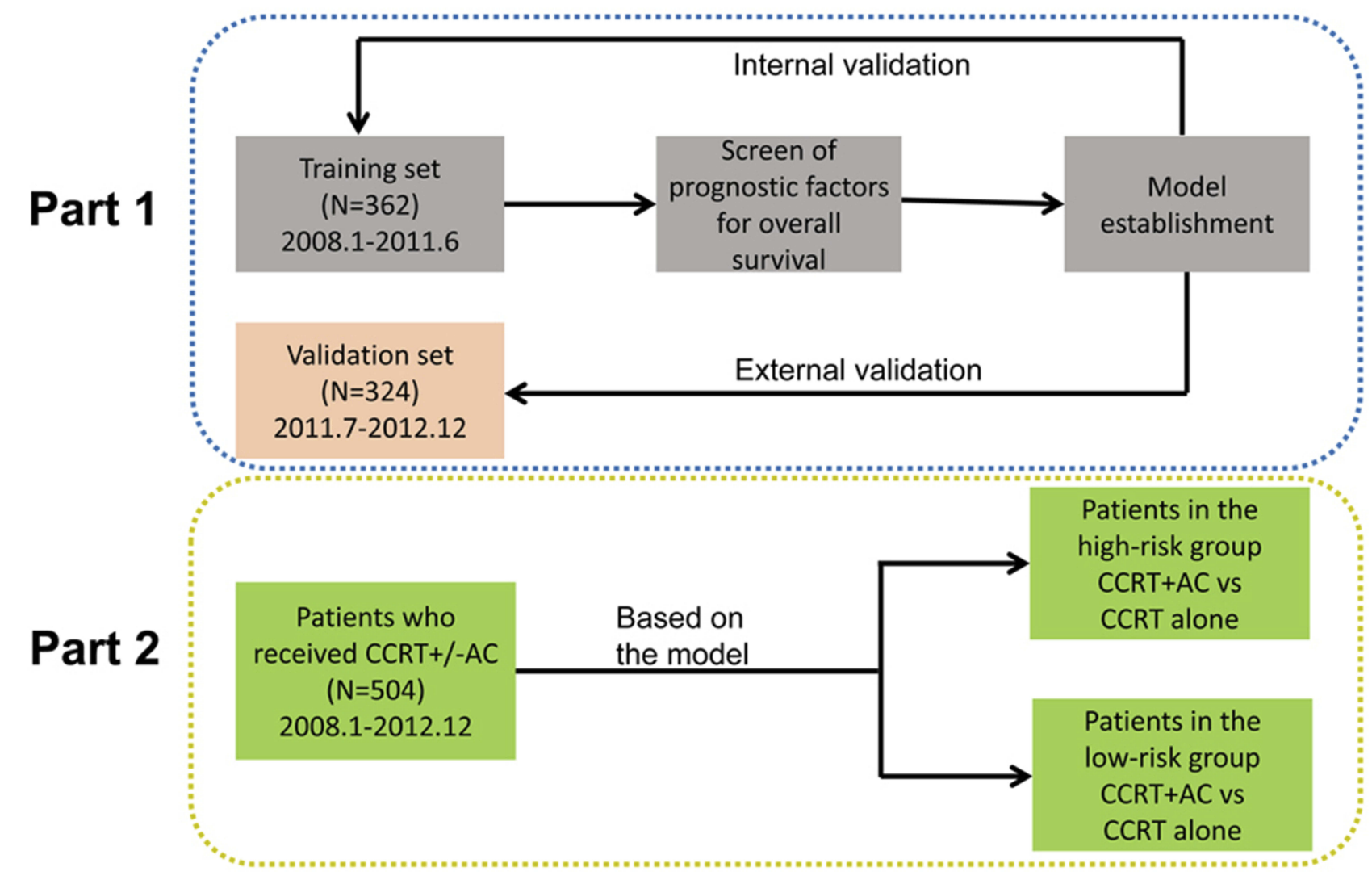

Figure I Study design. Part I: Construction of the prognostic model for overall survival to stratify NPC into high-risk and low-risk groups; Part 2: Application of the model to determine the role of the addition of AC to CCRT for the treatment of NPC.

Abbreviations: NPC, nasopharyngeal carcinoma; CCRT, concurrent chemoradiotherapy; AC, adjuvant chemotherapy. 
training data set for model development. The remaining 324 patients formed the validation data set. Patients who met the following criteria were enrolled in the study: (1) differentiated or undifferentiated non-keratinizing NPC (WHO classification); (2) without distant metastasis; and (3) treated with IMRT. All of the NPC cases were restaged accurately on the basis of the criteria of the 8th edition American Joint Committee on Cancer (AJCC) staging system by two radiologists who specialize in head and neck cancers. Patients with a previous malignancy, pregnancy or lactation, or severe infection or complications, such as unstable cardiac disease requiring treatment, were excluded.

\section{Treatment Strategies}

A detailed description of IMRT has been published previously. ${ }^{12}$ The prescribed dose was $68-74$ Gy for the primary tumour, 60-71 Gy for any involved cervical lymph nodes, 60-66 Gy for the high-risk region, and 54-60 Gy for the low-risk region in 30-32 fractions over a period of $6 \sim 7$ weeks. Patients with stage I disease received IMRT alone. For patients with stage II-IV disease, IMRT in combination with systemic chemotherapy of platinum-based regimens was administered. Concurrent chemotherapy comprised cisplatin alone $\left(100 \mathrm{mg} / \mathrm{m}^{2}\right)$ or cisplatin $\left(80 \mathrm{mg} / \mathrm{m}^{2}\right)$ on day 1 and 5 -fluorouracil (5-FU; $750 \mathrm{mg} / \mathrm{m}^{2} /$ day) by continuous intravenous infusion at $96 \mathrm{~h}$. The regimens of induction chemotherapy (IC) or AC comprised a combination of a platinum-based regimen with two or three drugs, including TPF (docetaxel $75 \mathrm{mg} / \mathrm{m}^{2}$, cisplatin $75 \mathrm{mg} / \mathrm{m}^{2}$, with 5-fluorouracil $750 \mathrm{mg} / \mathrm{m}^{2}$ for $96 \mathrm{~h}$ continuously; docetaxel $60 \mathrm{mg} / \mathrm{m}^{2}$, cisplatin $60 \mathrm{mg} / \mathrm{m}^{2}$, with 5 -fluorouracil $600 \mathrm{mg} / \mathrm{m}^{2}$ for $120 \mathrm{~h}$ continuously), PF (cisplatin $80 \mathrm{mg} / \mathrm{m}^{2}$ and 5 -fluorouracil $750 \mathrm{mg} / \mathrm{m}^{2}$ for $96 \mathrm{~h}$ continuously), and TP (docetaxel, with cisplatin, $75 \mathrm{mg} / \mathrm{m}^{2}$ on day 1).

\section{Follow-Up}

After completion of the treatments, the patients were followed up every 3 months during the first 2 years, every 6 months in the third to fifth years, and annually thereafter through clinic visits or telephone interviews. The information obtained was used to assess survival, relapse patterns, and distant metastasis incidence. Examinations included ultrasound scans of the liver and abdomen, chest X-rays or computed tomography (CT) scans, whole-body bone scans, CT or magnetic resonance imaging scans of the head and neck, and nasopharyngoscopy with or without biopsy.

Table I Characteristics of the Patients with Nasopharyngeal Carcinoma in the Training and Validation Sets

\begin{tabular}{|c|c|c|}
\hline Characteristics & $\begin{array}{l}\text { Training Set } \\
(\mathrm{N}=362)\end{array}$ & $\begin{array}{l}\text { Validation Set } \\
(\mathrm{N}=324)\end{array}$ \\
\hline \multicolumn{3}{|l|}{ Sex } \\
\hline Male & $266(73.5 \%)$ & 257 (79.3\%) \\
\hline Female & $96(26.5 \%)$ & 67 (20.7\%) \\
\hline Age, years ${ }^{\mathrm{a}}$ & $45(16-78)$ & $46(16-86)$ \\
\hline \multicolumn{3}{|l|}{ KPS } \\
\hline $70-80$ & I 58 (43.6\%) & III (34.3\%) \\
\hline $90-100$ & $204(56.4 \%)$ & $213(65.7 \%)$ \\
\hline \multicolumn{3}{|l|}{$\mathrm{T}$ classification ${ }^{\mathrm{b}}$} \\
\hline TI & $4 \mathrm{I}(\mathrm{II} .3 \%)$ & 18 (5.6\%) \\
\hline $\mathrm{T} 2$ & $116(32.0 \%)$ & 89 (27.5\%) \\
\hline T3 & 145 (40.1\%) & $134(4 \mid .4 \%)$ \\
\hline $\mathrm{T} 4$ & $60(16.6 \%)$ & $83(25.6 \%)$ \\
\hline \multicolumn{3}{|l|}{$N$ classification ${ }^{b}$} \\
\hline No & $38(10.5 \%)$ & 15 (4.6\%) \\
\hline NI & $128(35.4 \%)$ & II2 (34.6\%) \\
\hline N2 & 149 (4I.2\%) & 142 (43.8\%) \\
\hline N3 & 47 (I3.0\%) & $55(17.0 \%)$ \\
\hline \multicolumn{3}{|l|}{ Clinical stage ${ }^{b}$} \\
\hline I & 9 (2.5\%) & $3(0.9 \%)$ \\
\hline II & $76(21.0 \%)$ & $5 \mathrm{I}(\mathrm{I} 5.7 \%)$ \\
\hline III & 172 (47.5\%) & I 49 (46.0\%) \\
\hline IVA & $105(29.0 \%)$ & 121 (37.3\%) \\
\hline Neutrophil, k/cc ${ }^{a}$ & $3.94(0.74-13.47)$ & $4.1(1.38-14.32)$ \\
\hline Haemoglobin, $g / \mathrm{L}^{\mathrm{a}}$ & $136(79-182)$ & $|4|(74-\mid 85)$ \\
\hline Platelets, $\mathrm{k} / \mathrm{cc}^{\mathrm{a}}$ & $238.5(77-494)$ & $230(42-625)$ \\
\hline $\begin{array}{l}\text { Serum alkaline } \\
\text { phosphatase, } U / L^{\mathrm{a}}\end{array}$ & $54(11-156)$ & $63(23-176)$ \\
\hline $\begin{array}{l}\text { Serum lactate } \\
\text { dehydrogenase, } U / L^{\mathrm{a}}\end{array}$ & $172(|03-45|)$ & $174(102-447)$ \\
\hline Serum ferritin, $U / L^{a}$ & $25 I(9-74 I)$ & $278.5(7-726)$ \\
\hline Serum albumin, $g / L^{a}$ & $42.9(30.9-55.2)$ & $43.4(13.8-56)$ \\
\hline \multicolumn{3}{|l|}{ Treatment regimens } \\
\hline IMRT & 40 (II.0\%) & $30(9.3 \%)$ \\
\hline CCRT & 74 (20.4\%) & 101 (31.2\%) \\
\hline$C C R T+A C$ & $182(50.3 \%)$ & 147 (45.4\%) \\
\hline $\mathrm{IC}+\mathrm{CCRT}$ & $28(7.7 \%)$ & $19(5.9 \%)$ \\
\hline$I C+C C R T+A C$ & $27(7.5 \%)$ & $26(8.0 \%)$ \\
\hline IC+IMRT & 4 (I.I\%) & I (0.3\%) \\
\hline$I C+I M R T+A C$ & 7 (1.9\%) & $0(0.0 \%)$ \\
\hline
\end{tabular}

Notes: ${ }^{\text {aT }}$ The median and the range of values. ${ }^{\text {b}}$ The 8 th edition American Joint Committee on Cancer staging system. ${ }^{16,17}$

Abbreviations: IMRT, intensity modulated radiotherapy; CCRT, concurrent chemoradiotherapy; IC, induction chemotherapy; AC, adjuvant chemotherapy. 
Table 2 Univariate and Multivariate Analyses of Risk Factors for Death Among Patients in the Training Set

\begin{tabular}{|c|c|c|c|c|}
\hline \multirow[t]{2}{*}{ Factors } & \multicolumn{2}{|l|}{ Univariate Analysis } & \multicolumn{2}{|c|}{ Multivariate Analysis } \\
\hline & HR $(95 \% \mathrm{CI})$ & $\mathbf{P}$ & HR (95\% Cl) & $\mathbf{P}$ \\
\hline Sex (female vs male) & $0.642(0.389-1.059)$ & 0.080 & $0.937(0.537-1.636)$ & 0.820 \\
\hline T stage & $1.600(1.263-2.027)$ & $<0.001$ & $1.539(1.208-1.962)$ & $<0.001$ \\
\hline $\mathrm{N}$ stage & $1.459(1.149-1.853)$ & 0.015 & $1.564(1.208-2.024)$ & 0.001 \\
\hline KPS (70-80 vs 90-100) & $1.216(0.8 \mid 2-1.81 \mathrm{I})$ & 0.335 & & \\
\hline Age, years (continuous) & $1.039(1.021-1.058)$ & $<0.001$ & $1.038(1.018-1.058)$ & $<0.001$ \\
\hline Neutrophil, k/cc (continuous) & $1.050(0.942-1.170)$ & 0.382 & & \\
\hline Haemoglobin, g/L (continuous) & $0.997(0.985-1.010)$ & 0.675 & & \\
\hline Platelets, k/cc (continuous) & $1.000(0.997-1.003)$ & 0.884 & & \\
\hline Alkaline phosphatase, U/L (continuous) & $1.018(1.010-1.027)$ & $<0.001$ & $1.014(1.005-1.023)$ & 0.002 \\
\hline Lactate dehydrogenase, U/L (continuous) & $1.003(0.999-1.006)$ & 0.140 & & \\
\hline Serum ferritin, mg/L (continuous) & $1.001(1.000-1.002)$ & 0.094 & $1.000(0.999-1.002)$ & 0.563 \\
\hline Serum albumin, $g / L$ (continuous) & $0.926(0.882-0.972)$ & 0.002 & $0.965(0.918-1.015)$ & 0.164 \\
\hline
\end{tabular}

Abbreviations: $\mathrm{HR}$, hazard ratio; $\mathrm{Cl}$, confidence interval.

\section{Statistical Analysis \\ Model Construction}

Survival analyses were performed with SPSS software, version 16.0 (SPSS Inc., Chicago, IL). Age, sex, T stage, $\mathrm{N}$ stage, Karnofsky performance score (KPS), neutrophil count, haemoglobin, platelet count, serum ALP, serum LDH, serum ferritin, and serum albumin were analysed for associations with OS. Univariate analyses for OS were estimated with the Log rank test for categorical variables; the Cox proportional test was utilized for continuous variables. Predictors with P-values $<0.1$ in the univariate analysis were entered into multivariate analysis to validate their significance with the Cox proportional hazards model by using the backward stepwise algorithm. Covariates with a univariate $\mathrm{P}<0.05$ were included in the multivariate model for building the prognostic index (PI) by using an iterative process of backward selection. A PI scoring system for OS was constructed based on the weighting (derived by the b-coefficient of the respective log [adjusted hazard ratios (AHRs)]) of the significant covariates in the training cohort. The PI scores were then dichotomized for OS by using the optimal cut-off values determined by the "surv_cutpoint" function of the "survminer" $\mathrm{R}$ package ${ }^{13}$ in R version 3.5.1 (http://www.r-project.org/). The patients were then dichotomized into low-risk and high-risk subgroups in the training cohort.

\section{Evaluation of Discrimination and Calibration}

The discrimination of the prognostic model was assessed by calculating the Harrell concordance index (C-index) by using the method by Newson (11) with implementation of the package "rms" in $\mathrm{R}$ software. As a means of internal validation, bootstrapping was applied to correct the c-index for optimism with 1000 repetitions from the training database. A Kaplan-Meier survival curve was used to compare OS, distant metastasis-free survival (DMFS), loco-regional failure-free survival (LFFS), and failure-free survival (FFS) between the low- and high-risk groups in the training and validation sets. In addition, calibration curves were assessed graphically by plotting the observed rates against the PIpredicted probabilities via a bootstrap method with 1000 resamples.

\section{Comparison Between CCRT-AC and CCRT Alone}

Survival analyses for OS and DMFS between the CCRT-AC and CCRT alone groups were estimated with the Log rank test for the high- and low-risk groups. Patients who received induction chemotherapy were not included in this part of the analysis. If key baseline characteristics were not balanced between the two groups, the propensity score-matching

Table 3 Constructed Prognostic Index to Predict Overall Survival for Patients with Nasopharyngeal Carcinoma

\begin{tabular}{|l|l|l|}
\hline Covariate & $\boldsymbol{\beta}[\mathrm{HR}=\operatorname{exp(\beta )]}$ & Score \\
\hline T stage & 0.4586 & $45.86 \times(\mathrm{T}-\mathrm{I})$ \\
$\mathrm{N}$ stage & 0.4403 & $44.03 \times \mathrm{N}$ \\
Age, years & 0.0419 & $4.19 \times$ age $(\mathrm{y})$ \\
Alkaline phosphatase, U/L & 0.0149 & $1.49 \times$ Alkaline phosphatase (U/L) \\
Total computed score & & \\
and risk stratification & & \\
Low risk & & $\leq 407.96$ \\
High risk & & $>407.96$ \\
\hline
\end{tabular}

Abbreviation: $H R$, hazard ratio. 
(PSM) method was used to match patients between these two groups considering clinical stage with the implementation of the "nonrandom" package in R software (version 3.5.1). ${ }^{14,15}$ values $<0.05$ were considered statistically significant.

The study design flowchart is presented in Figure 1.

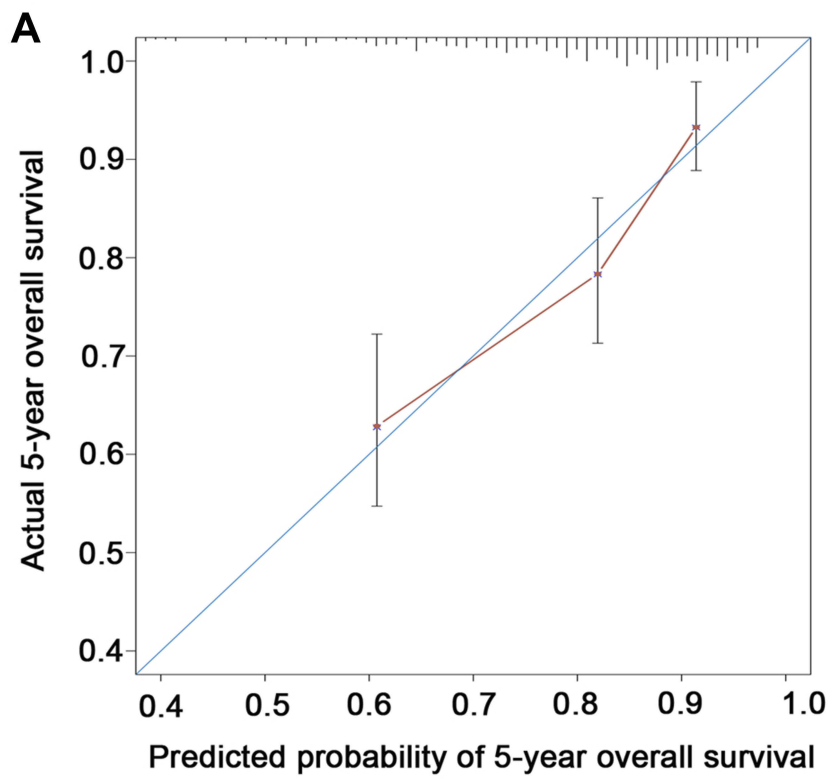

\section{Results}

\section{Construction of a PI for OS in} Nasopharyngeal Carcinoma

The clinical characteristics of the patients in the training and validation cohorts are listed in Table 1 . To build the

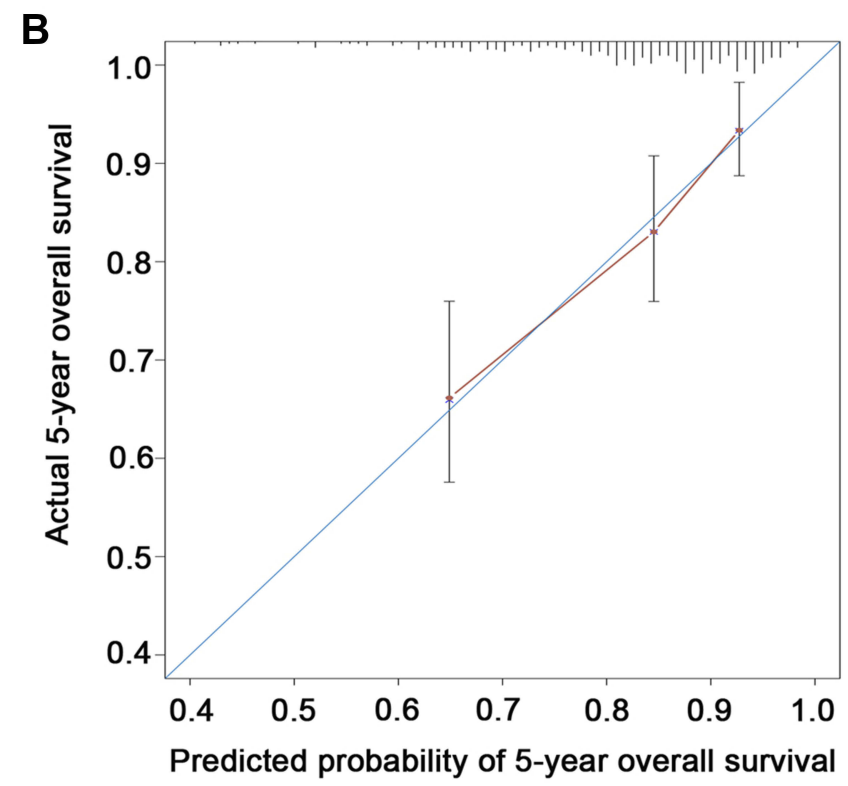

C



Figure 2 Calibration and discrimination of the prognostic model. Calibration plots of survival probabilities at 5 years for patients with nasopharyngeal carcinoma in the training set $(\mathbf{A})$ and the validation set $(\mathbf{B})$; a cut-off of 407.96 for dividing patients into high-risk and low-risk groups in the training set by using maximally selected rank statistics $(\mathbf{C})$. 
prognostic model, we first tested the covariates listed in Table 2 for their association with OS in the training cohort. According to the results of univariate analysis, sex, $\mathrm{T}$ classification, $\mathrm{N}$ classification, age, ALP, serum ferritin and serum albumin were included in the multivariate analysis. The significant predictors of OS in multivariate analysis were T stage (AHR 1.539, 95\% CI 1.208-1.962), N stage (AHR 1.564, 95\% CI 1.208-2.024), age (AHR 1.038, 95\% CI 1.018-1.058), and ALP (AHR 1.014, 95\% CI 1.005-1.023). We then constructed a PI based on weighting (derived by the b-coefficient of the respective $\log$ [AHRs]) of these four significant covariates in the training cohort (Table 3 ).

$$
\begin{aligned}
\mathrm{PI}= & 45.86 \times(\mathrm{T}-1)+44.03 \times \mathrm{N}+4.19 \times \operatorname{age}(\mathrm{y}) \\
& +1.49 \times \operatorname{ALP}(\mathrm{U} / \mathrm{L})
\end{aligned}
$$

The model yielded a C-index of 0.718 (95\% CI 0.660-0.777 ) for OS, which was superior to the $\mathrm{C}$-index of 0.638 (95\% CI 0.583-0.692) for OS when using the 8th AJCC edition overall stage. Calibration plots showed good agreement between the predicted probabilities and the actual observations of 5-year OS (Figure 2A).

By using the "surv_cutpoint" function of the "survminer" R package, we were able to define low-risk and highrisk subgroups based on a cut-off of 407.96 (Figure 2C). According to survival analysis, there were significant differences in OS (HR 5.210, 95\% CI 3.118-8.704; $\mathrm{P}<$ 0.001), LFFS (HR 3.050, 95\% CI 1.651-5.635; P < 0.001), DMFS (HR 3.132, 95\% CI 1.772-5.536; $\mathrm{P}<0.001$ ), and FFS (HR 3.199, 95\% CI 2.105-4.861; P < 0.001) between the high-risk and low-risk groups in the training set (details are provided in Figure $3 \mathrm{~A}-\mathrm{D}$ ).

\section{Validation of the PI for OS Prediction}

To validate the predictive accuracy of our PI for OS in NPC, internal validation using the bootstrapping technique with 1000 repetitions resulted in a C-index of 0.719 (95\% CI 0.677-0.760), which was significantly higher than the $0.638(95 \%$ CI $0.597-0.678)$ obtained by applying the 8th AJCC edition $(\mathrm{P}<0.001)$. In addition, we conducted external validation in a later cohort of 324 consecutively treated patients. The same model yielded a C-index of 0.707 (95\% CI 0.634-0.780) for OS, which was superior to the clinical stage C-index of 0.649 (95\% CI 0.582-0.716) in the validation cohort. Moreover, calibration plots revealed superb agreement between the predicted probabilities and the actual observations of 5-year OS (Figure 2B).

By using the same PI score cut-off of 407.96, we stratified a high-risk subgroup with a significantly inferior OS compared with a low-risk subgroup (HR
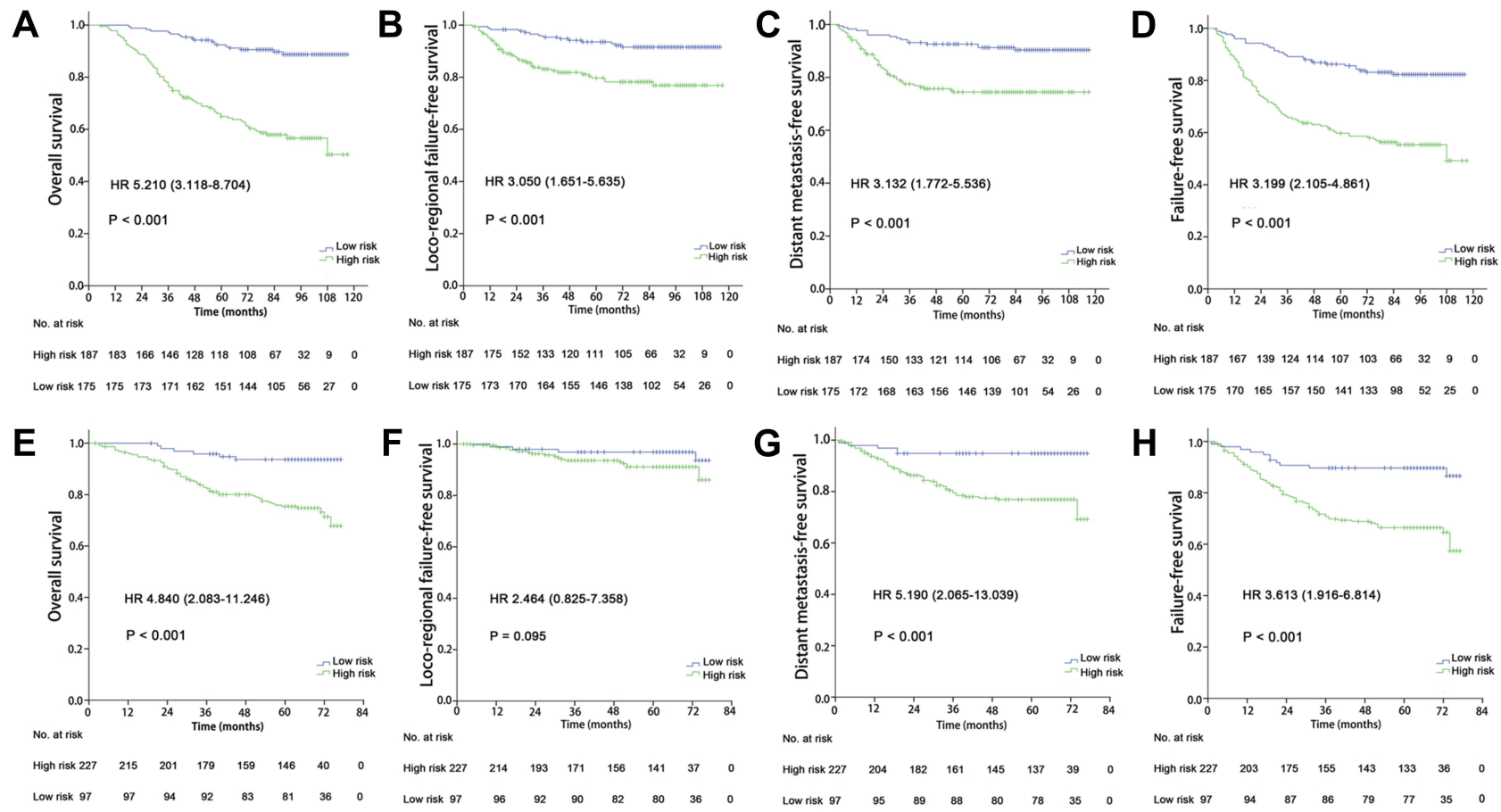

Figure 3 Kaplan-Meier curves of overall survival, loco-regional failure-free survival, distant metastasis-free survival, and failure-free survival between the high-risk and lowrisk groups of nasopharyngeal carcinoma patients in the training (A-D) and validation (E-H) sets. 
4.840, 95\% CI 2.083-11.246; $\mathrm{P}<0.001$ ). Additionally, contrast, no significant difference in loco-regional patients in the high-risk group had a higher risk of recurrence was found between the two groups (HR distant metastasis (HR 5.190, 95\% CI 2.065-13.039; 2.464, 95\% CI 0.825-7.358; P = 0.095) (details are $\mathrm{P}<0.001)$ than those in the low-risk group. In shown in Figure 3E-H).

Table 4 Characteristics of Nasopharyngeal Carcinoma Patients Treated with CCRT \pm AC in the High-Risk Group

\begin{tabular}{|c|c|c|c|c|c|c|}
\hline \multirow[t]{2}{*}{ Characteristics } & \multicolumn{2}{|l|}{ All Cases } & \multirow[t]{2}{*}{$\mathbf{P}$} & \multicolumn{2}{|l|}{ Matched Cases } & \multirow[t]{2}{*}{$\mathbf{P}$} \\
\hline & CCRT-AC $(\mathrm{N}=206)$ & CCRT $(\mathrm{N}=101)$ & & CCRT-AC $(\mathrm{N}=\mid 77)$ & CCRT $(\mathrm{N}=89)$ & \\
\hline Sex & & & 0.310 & & & 0.227 \\
\hline Male & $|7|(83.0 \%)$ & 79 (78.2\%) & & I 48 (83.6\%) & 69 (77.5\%) & \\
\hline Female & $35(17.0 \%)$ & $22(21.8 \%)$ & & $29(16.4 \%)$ & $20(22.5 \%)$ & \\
\hline Age, years & & & 0.869 & & & 0.096 \\
\hline Median (range) & $48(16-69)$ & $52(27-70)$ & & $48(16-69)$ & $52(27-70)$ & \\
\hline KPS & & & 0.101 & & & 0.051 \\
\hline $70-80$ & $100(48.5 \%)$ & $39(38.6 \%)$ & & $90(50.8 \%)$ & $34(38.2 \%)$ & \\
\hline $90-100$ & $106(51.5 \%)$ & 62 (6I.4\%) & & 87 (49.2\%) & $55(61.8 \%)$ & \\
\hline T classification ${ }^{\mathrm{a}}$ & & & 0.052 & & & 0.140 \\
\hline TI & $3(1.5 \%)$ & $3(3.0 \%)$ & & $3(1.7 \%)$ & $2(2.2 \%)$ & \\
\hline $\mathrm{T} 2$ & $21(10.2 \%)$ & $2 \mathrm{I}(20.8 \%)$ & & $19(10.7 \%)$ & 17 (I9.2\%) & \\
\hline T3 & II 4 (55.3\%) & 46 (45.5\%) & & 101 (57.1\%) & 39 (43.8\%) & \\
\hline $\mathrm{T} 4$ & $68(33.0 \%)$ & $31(30.7 \%)$ & & 54 (30.5\%) & $31(34.8)$ & \\
\hline $\mathrm{N}$ classification ${ }^{\mathrm{a}}$ & & & 0.119 & & & 0.733 \\
\hline No & $5(2.4 \%)$ & 4 (4.0\%) & & $5(2.8 \%)$ & $3(3.4 \%)$ & \\
\hline $\mathrm{NI}$ & $52(25.2 \%)$ & $36(35.6 \%)$ & & 47 (26.6\%) & $29(32.6 \%)$ & \\
\hline N2 & $106(51.5 \%)$ & 45 (44.6\%) & & 87 (49.2\%) & $4 \mid(46.1 \%)$ & \\
\hline N3 & 43 (20.9\%) & $16(15.8 \%)$ & & $38(21.4 \%)$ & $16(18.0 \%)$ & \\
\hline Clinical stage ${ }^{a}$ & & & 0.004 & & & 0.069 \\
\hline II & I (0.5\%) & 7 (6.9\%) & & I (0.6\%) & 4 (4.5\%) & \\
\hline III & 102 (49.5\%) & 49 (48.5\%) & & $90(50.8 \%)$ & 40 (44.9\%) & \\
\hline IVA & $103(50.0 \%)$ & 45 (44.6\%) & & $86(48.6 \%)$ & $45(50.6 \%)$ & \\
\hline CC regimens & & & 0.842 & & & \\
\hline DDP & 191 (92.7\%) & 93 (92.1\%) & & $163(92.1 \%)$ & 84 (94.4\%) & \\
\hline PF & 15 (7.3\%) & 8 (7.9\%) & & 14 (7.9\%) & $5(5.6 \%)$ & \\
\hline CC cycles & & & $<0.001$ & & & 0.551 \\
\hline 1 & 10 (4.9\%) & 19 (I8.8\%) & & $10(5.6 \%)$ & $8(9 \%)$ & \\
\hline 2 & 106 (51.5\%) & 45 (44.6\%) & & 95 (53.7\%) & 44 (49.4\%) & \\
\hline 3 & $90(43.6 \%)$ & $37(36.6 \%)$ & & 72 (40.7\%) & 37 (4I.6\%) & \\
\hline$A C$ regimens & & & & & & \\
\hline TPF & I (0.5\%) & I & & I (0.6\%) & I & \\
\hline TP & $20(9.7 \%)$ & I & & 20 (II.3\%) & I & \\
\hline PF & I 85 (89.8\%) & I & & I56 (88.1\%) & I & \\
\hline AC cycles & & & & & & \\
\hline 1 & $54(26.2 \%)$ & I & & 46 (26\%) & I & \\
\hline 2 & 116 (56.3\%) & 1 & & $100(56.5 \%)$ & I & \\
\hline 3 & 36 (17.5\%) & 1 & & 31 (I7.5\%) & 1 & \\
\hline
\end{tabular}

Notes: ${ }^{a}$ The 8 th edition American Joint Committee on Cancer staging system..$^{16,17}$

Abbreviations: CCRT, concurrent chemoradiotherapy; CC, concurrent chemotherapy; AC, adjuvant chemotherapy; DDP, cisplatin; PF, cisplatin and fluorouracil; TP, docetaxel and cisplatin; TPF, docetaxel, cisplatin and fluorouracil. 


\section{CCRT-AC versus CCRT Alone in}

\section{Nasopharyngeal Carcinoma}

According to the model, 307 patients who received CCRT with or without $\mathrm{AC}$ were categorized into the high-risk group (see Table 4). Compared to CCRT alone, a significant benefit of OS was found with the addition of AC (HR, 0.620, 95\% CI 0.408-0.941; P $=0.023$ ) (Figure 4A). However, a higher percentage of IVA diseases and a higher percentage of 2-3 cycles of concurrent chemotherapy were observed in the CCRT-AC group. After PSM (2:1) using these two factors, 266 patients were selected; of these, 177 were in the CCRT-AC group and 89 in the CCRT alone group (Table 4). A borderline significant difference in OS was observed in favour of the CCRT-AC group (HR, 0.648, 95\% CI 0.414-1.012; $\mathrm{P}=$ 0.054) (Figure 4B), though no significant difference in DMFS was found between the two groups before and after PSM (Figure 4C and D).

In the low-risk group, 123 patients received CCRT-AC, and 74 received CCRT alone (details are shown in Table 5). A borderline significant difference in OS (HR, 3.915, 95\% $\mathrm{CI}, 0.882$ to 17.376 ; $\mathrm{P}=0.053$ ) was found, with better outcomes for the CCRT alone group (Figure 4E). Moreover, addition of adjuvant chemotherapy increased the risk of distant metastasis in low-risk patients (HR, 4.222, 95\% CI, 0.959 to $18.585 ; \mathrm{P}=0.038$ ) (Figure $4 \mathrm{G}$ ). However, the baseline characteristics of the patients between the two groups were unbalanced, including the $\mathrm{N}$ category and clinical stage. After PSM (2:1) by clinical stage, 164 patients were selected; of these, 109 were in the CCRT-AC group and 55 in the CCRT alone group (Table 5). Although no significant difference was observed between the two groups in terms of OS (HR, 5.546, 95\% CI, 0.716 to 42.986; $\mathrm{P}=0.065$ ) (Figure 4F), significant differences were found in terms of DMFS (HR, $6.028,95 \% \mathrm{CI}, 0.783$ to $46.375 ; \mathrm{P}=0.049$ ), showing better outcomes in the CCRT alone group (Figure 4H).

\section{Discussion}

In the present study, we constructed a prognostic model for OS among patients with NPC that was able to stratify high-risk and low-risk groups. This model combined clinical characteristics and experimental factors and showed better discrimination of OS than the overall stage of the 8th edition AJCC staging system in both training and validation sets.

Clinically, patients with the same TNM stage may have different mortality risk. Therefore, other factors may need to be incorporated into prognostic models. The present study showed that $\mathrm{T}$ stage, $\mathrm{N}$ stage, age, and ALP are significant
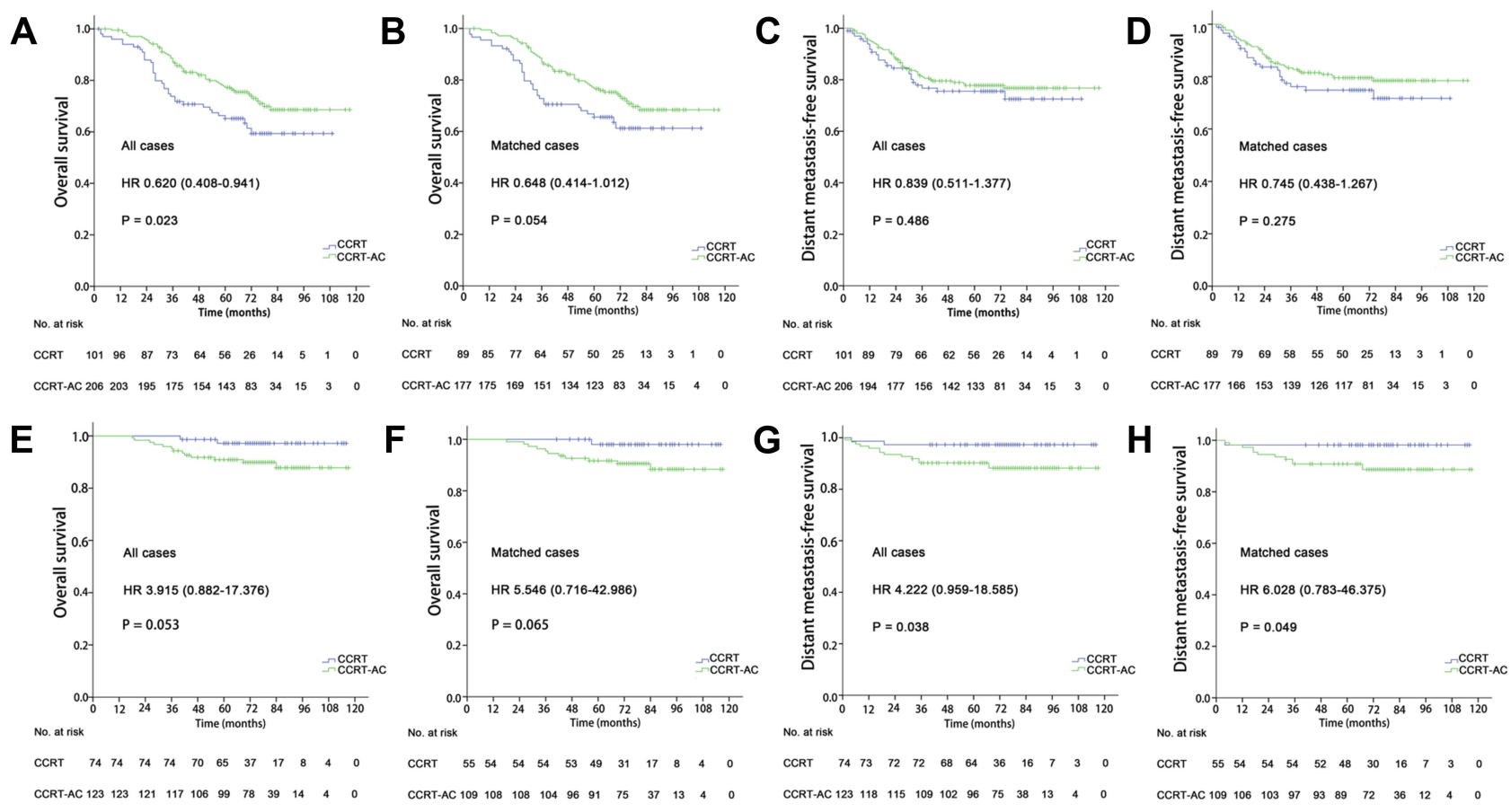

Figure 4 Kaplan-Meier curves of overall survival and distant metastasis-free survival between the CCRT-AC and CCRT alone groups of high-risk (A-D) and low-risk (E-H) patients.

Abbreviations: CCRT, concurrent chemoradiotherapy; AC, adjuvant chemotherapy. 
prognostic factors for NPC. Several studies have reported that these four factors are closely related to OS, loco-regional recurrence, and distant metastasis. ${ }^{16-18}$ Yang and colleagues demonstrated that both the $\mathrm{T}$ and $\mathrm{N}$ category classifications of the 8 th edition are independent prognostic factors for OS. ${ }^{16}$
The higher is the T stage or N stage, the poorer is the prognosis. Age and pretreatment ALP have also been shown to be prognostic factors for NPC. In the studies by Huang et $\mathrm{al}^{19}$ and $\mathrm{Xu}$ et $\mathrm{al},{ }^{20}$ the older age groups had a significant negative influence on OS. Furthermore, Li and colleagues found that

Table 5 Characteristics of Nasopharyngeal Carcinoma Patients Treated with Concurrent CCRT \pm AC in the Low-Risk Group

\begin{tabular}{|c|c|c|c|c|c|c|}
\hline \multirow[t]{2}{*}{ Characteristics } & \multicolumn{2}{|l|}{ All Patients } & \multirow[t]{2}{*}{$\mathbf{P}$} & \multicolumn{2}{|l|}{ Matched Patients } & \multirow[t]{2}{*}{$\mathbf{P}$} \\
\hline & CCRT-AC $(\mathrm{N}=\mid 23)$ & CCRT $(\mathrm{N}=74)$ & & CCRT-AC $(\mathrm{N}=109)$ & CCRT $(\mathrm{N}=55)$ & \\
\hline $\begin{array}{l}\text { Sex } \\
\qquad \text { Male } \\
\text { Female }\end{array}$ & $\begin{array}{l}84(68.3 \%) \\
39(31.7 \%)\end{array}$ & $\begin{array}{l}55(74.3 \%) \\
19(25.7 \%)\end{array}$ & 0.368 & $\begin{array}{l}76(69.7 \%) \\
33(30.3 \%)\end{array}$ & $\begin{array}{l}38(69.1 \%) \\
17(30.9 \%)\end{array}$ & 0.934 \\
\hline $\begin{array}{l}\text { Age, years } \\
\text { Median (range) }\end{array}$ & $40(18-56)$ & $4 I(18-55)$ & 0.392 & $40(18-56)$ & $40(18-55)$ & 0.889 \\
\hline $\begin{array}{l}\text { KPS } \\
\qquad \begin{array}{l}70-80 \\
90-100\end{array}\end{array}$ & $\begin{array}{l}42(34.1 \%) \\
81(65.9 \%)\end{array}$ & $\begin{array}{l}16(21.6 \%) \\
58(78.4 \%)\end{array}$ & 0.062 & $\begin{array}{l}37(33.9 \%) \\
72(66.1 \%)\end{array}$ & $\begin{array}{l}13(23.6 \%) \\
42(76.4 \%)\end{array}$ & 0.176 \\
\hline $\begin{array}{l}\text { T classification } \\
\text { T1 } \\
\text { T2 } \\
\text { T3 } \\
\text { T4 }\end{array}$ & $\begin{array}{l}17(13.8 \%) \\
60(48.8 \%) \\
42(34.1 \%) \\
4(3.3 \%)\end{array}$ & $\begin{array}{l}10(13.5 \%) \\
47(63.5 \%) \\
15(20.3 \%) \\
2(2.7 \%)\end{array}$ & 0.174 & $\begin{array}{l}13(11.9 \%) \\
56(51.4 \%) \\
40(36.7 \%) \\
0(0.0 \%)\end{array}$ & $\begin{array}{l}7(12.7 \%) \\
31(56.4 \%) \\
15(27.3 \%) \\
2(3.6 \%)\end{array}$ & 0.163 \\
\hline $\begin{array}{l}\text { N classification } \\
\text { No } \\
\text { NI } \\
\text { N2 } \\
\text { N3 }\end{array}$ & $\begin{array}{l}10(8.1 \%) \\
61(49.6 \%) \\
42(34.1 \%) \\
10(8.1 \%)\end{array}$ & $\begin{array}{l}6(8.1 \%) \\
46(62.2 \%) \\
22(29.7 \%) \\
0(0.0 \%)\end{array}$ & 0.054 & $\begin{array}{l}8(7.3 \%) \\
59(54.1 \%) \\
38(34.9 \%) \\
4(3.7 \%)\end{array}$ & $\begin{array}{l}4(7.3 \%) \\
29(52.7 \%) \\
22(40.0 \%) \\
0(0.0 \%)\end{array}$ & 0.513 \\
\hline $\begin{array}{l}\text { Clinical stage }^{\mathrm{a}} \\
\text { II } \\
\text { III } \\
\text { IVA }\end{array}$ & $\begin{array}{l}41 \text { (33.3\%) } \\
68(55.3 \%) \\
14(11.4 \%)\end{array}$ & $\begin{array}{l}40(54.1 \%) \\
32(43.2 \%) \\
2(2.7 \%)\end{array}$ & 0.005 & $\begin{array}{l}4 \mathrm{I}(37.6 \%) \\
64(58.7 \%) \\
4(3.7 \%)\end{array}$ & $\begin{array}{l}21(38.2 \%) \\
32(58.2 \%) \\
2(3.6 \%)\end{array}$ & 0.998 \\
\hline $\begin{array}{l}\text { CC regimens } \\
\text { DDP } \\
\text { PF }\end{array}$ & $\begin{array}{l}115(93.5 \%) \\
8(6.5 \%)\end{array}$ & $\begin{array}{l}68(91.9 \%) \\
6(8.1 \%)\end{array}$ & 0.671 & $\begin{array}{l}101(92.7 \%) \\
8(7.3 \%)\end{array}$ & $\begin{array}{l}49(89.1 \%) \\
6(10.9 \%)\end{array}$ & 0.44 \\
\hline $\begin{array}{c}\text { CC cycles } \\
1 \\
2 \\
3\end{array}$ & $\begin{array}{l}8(6.5 \%) \\
68(55.3 \%) \\
47(38.2 \%)\end{array}$ & $\begin{array}{l}6(8.1 \%) \\
31(41.9 \%) \\
37(50.0 \%)\end{array}$ & 0.19 & $\begin{array}{l}8(7.3 \%) \\
61(56.0 \%) \\
40(36.7 \%)\end{array}$ & $\begin{array}{l}6(10.9 \%) \\
26(47.3 \%) \\
23(41.8 \%)\end{array}$ & 0.518 \\
\hline $\begin{array}{l}\text { AC regimens } \\
\text { TP } \\
\text { PF }\end{array}$ & $\begin{array}{l}10(8.1 \%) \\
113(91.9 \%)\end{array}$ & $\begin{array}{l}1 \\
1\end{array}$ & & $\begin{array}{l}10(9.2 \%) \\
99(90.8 \%)\end{array}$ & $\begin{array}{l}1 \\
1\end{array}$ & \\
\hline $\begin{array}{l}\text { AC cycles } \\
\qquad \begin{array}{l}1 \\
2 \\
3\end{array}\end{array}$ & $\begin{array}{l}31 \text { ( } 25.2 \%) \\
72(58.5 \%) \\
20(16.3 \%)\end{array}$ & $\begin{array}{l}1 \\
1 \\
1\end{array}$ & & $\begin{array}{l}27(24.8 \%) \\
63(57.8 \%) \\
19(17.4 \%)\end{array}$ & $\begin{array}{l}1 \\
1 \\
1\end{array}$ & \\
\hline
\end{tabular}

Notes: ${ }^{\text {TThe }}$ 8th edition American Joint Committee on Cancer staging system. ${ }^{16,17}$

Abbreviations: CCRT, concurrent chemoradiotherapy; CC, concurrent chemotherapy; AC, adjuvant chemotherapy; DDP, cisplatin; PF, cisplatin and fluorouracil; TP, docetaxel and cisplatin. 
patients with higher ALP levels had poorer OS and LFFS than did those with a normal ALP level. ${ }^{8}$ Remarkably, the PI for OS was related to a significantly higher $\mathrm{C}$-index than the overall stage of the 8th edition AJCC staging system in both our training and validation sets. In addition, patients in the highrisk group had worse survival both with regard to distant metastasis and loco-regional recurrence in the training and validation sets, which indicates that more aggressive therapy regimens should be utilized for patients in high-risk groups.

In this cohort, patients in the high-risk group achieved survival benefits from AC. A series of studies has also demonstrated the importance of the addition of $\mathrm{AC}$ to reducing distant failure and improving OS in high-risk NPC patients with N3 disease, skull base destruction/intracranial invasion plus multiple node metastasis, or persistently detectable pEBV DNA after curative radiation therapy plus induction/concurrent chemotherapy. ${ }^{21,22}$ Moreover, we observed that the addition of adjuvant chemotherapy increased the risk of metastasis in low-risk patients. Similar results regarding the negative role of adjuvant chemotherapy were observed by Skillington et al, who reported that the addition of adjuvant chemotherapy to a radiotherapy regimen was associated with worse overall survival in patients with p16-positive oropharyngeal squamous cell carcinoma (adjusted HR, 1.46; 95\% CI, 0.91-2.33). ${ }^{23}$ During adjuvant chemotherapy, a cohort of patients with NPC experienced grade 3-4 dysphagia, nausea, and vomiting, ${ }^{24,25}$ leading to weight loss and nutritional deficiency. Indeed, poor nutritional status has a negative influence on overall survival and distant metastasis-free survival in NPC patients, ${ }^{26-28}$ which may correlate with the negative impacts of adjuvant chemotherapy observed in the present study. In addition, excessive chemotherapy for certain patients may lead to a loss of immune cells, which might result in worse survival. $^{29,30}$ The potential reasons why adjuvant chemotherapy acts as a double-edged sword in the treatment of NPC need to be explored in the future.

There were several limitations to our study. First, the training and validation sets were from the same institutes, which may reduce the level of evidence. Nonetheless, the two sets were from different periods, which might help minimize the potential negative impact. Second, due to the properties of retrospective studies, selection bias may have occurred because patients were included only if they met specific selection criteria. Third, recent studies have shown the important role of EBV-DNA in the NPC diagnosis, prognosis prediction, and decision making regarding whether AC should be added. ${ }^{20,31}$ Regardless, these relevant data are not available for all patients; therefore, we did not include this factor in the analysis.

In conclusion, the prognostic model for OS in the present study had both good discrimination and calibration and successfully stratified NPC patients into high-risk and low-risk groups. The addition of adjuvant chemotherapy might be a double-edged sword, bringing survival benefit to high-risk patients but a greater risk of distant metastasis to low-risk patients. Whether this prognostic model can be applied in other populations requires further study. Moreover, more prospective evidence from multiple centres is needed to confirm the influence of the addition of AC to CCRT on high-risk and low-risk patients.

\section{Ethics Statement}

The Ethics Committee of Guangxi Medical University Cancer Hospital approved the study protocol, in accordance with the guidelines of the Helsinki Declaration. Patient consent to review their medical records was not applicable due to the retrospective nature of the study, and the research involved no more than minimal risk to the participants. The data were anonymously analysed, and all the participants' personal information is confidential.

\section{Acknowledgments}

This work was sponsored by grants from the National Natural Science Foundation of China (No. 81760544), Key R\&D Program of Guangxi (GuiKe AB18221007), the Basic Ability Enhancement Project of Young Teachers in Guangxi Zhuang Autonomous Region (No. 2017KY0114), the Youth Science Foundation of Guangxi Medical University (No. GXMUYSF201515), and the International Communication of Guangxi Medical University Graduate Education in 2018. The funders had no role in the study design, data collection and analysis, decision to publish, or preparation of the manuscript.

\section{Author Contributions}

All authors made substantial contributions to the conception and design, acquisition of data, or analysis and interpretation of data; participated in drafting the article or revising it critically for important intellectual content; gave final approval of the version to be published; and agreed to be accountable for all aspects of the work. 


\section{Disclosure}

The authors report no conflicts of interest in this work.

\section{References}

1. Bray F, Ferlay J, Soerjomataram I, Siegel RL, Torre LA, Jemal A. Global cancer statistics 2018: GLOBOCAN estimates of incidence and mortality worldwide for 36 cancers in 185 countries. CA Cancer J Clin. 2018;68(6):394-424. doi:10.3322/caac.v68.6

2. Ng WT, Tung SY, Lee V, et al. Concurrent-adjuvant chemoradiation therapy for stage III-IVB nasopharyngeal carcinoma-exploration for achieving optimal 10-year therapeutic ratio. Int J Radiat Oncol Biol Phys. 2018;101(5):1078-1086. doi:10.1016/j.ijrobp.2018.04.069

3. Ribassin-Majed L, Marguet S, Lee AWM, et al. What is the best treatment of locally advanced nasopharyngeal carcinoma? An individual patient data network meta-analysis. J Clin Oncol. 2017;35 (5):498-505. doi:10.1200/JCO.2016.67.4119

4. Sun Y, Li WF, Chen NY, et al. Induction chemotherapy plus concurrent chemoradiotherapy versus concurrent chemoradiotherapy alone in locoregionally advanced nasopharyngeal carcinoma: a Phase 3, multicentre, randomised controlled trial. Lancet Oncol. 2016;17(11):1509-1520. doi:10.1016/S1470-2045(16)30410-7

5. Oei RW, Ye L, Kong F, et al. Pre-treatment serum lactate dehydrogenase is predictive of survival in patients with nasopharyngeal carcinoma undergoing intensity-modulated radiotherapy. $J$ Cancer. 2018;9(1):54-63. doi:10.7150/jca.22190

6. Su Z, Mao YP, OuYang PY, Tang J, Lan XW, Xie FY. Leucopenia and treatment efficacy in advanced nasopharyngeal carcinoma. $B M C$ Cancer. 2015;15:429. doi:10.1186/s12885-015-1442-3

7. Liang XX, Li Q, Su Z, et al. Significant prognostic impact of chemoradiotherapy-induced hemoglobin decrease on treatment outcomes of nasopharyngeal carcinoma. $J$ Cancer. 2015;6(6):502-510. doi: $10.7150 /$ jca. 11403

8. Li G, Gao J, Tao YL, et al. Increased pretreatment levels of serum LDH and ALP as poor prognostic factors for nasopharyngeal carcinoma. Chin J Cancer. 2012;31(4):197-206. doi:10.5732/ cjc.011.10283

9. Chen L, Hu CS, Chen XZ, et al. Adjuvant chemotherapy in patients with locoregionally advanced nasopharyngeal carcinoma: long-term results of a phase 3 multicentre randomised controlled trial. Eur J Cancer. 2017;75:150-158. doi:10.1016/j.ejca.2017.01.002

10. Liu LT, Chen QY, Tang LQ, et al. Neoadjuvant or adjuvant chemotherapy plus concurrent CRT versus concurrent CRT alone in the treatment of nasopharyngeal carcinoma: a study based on EBV DNA. $J$ Natl Compr Canc Netw. 2019;17(6):703-710. doi:10.6004/ jncen.2018.7270

11. Kim YS, Ahn YC, Lee CG, et al. The role of adjuvant chemotherapy after definitive chemoradiation therapy in locoregionally advanced nasopharyngeal carcinoma in a non-endemic area: multi-institutional retrospective study using propensity score matching analysis. Int J Radiat Oncol Biol Phys. 2018;100(5):1326. doi:10.1016/j. ijrobp.2017.12.054

12. Liang Z, Zhu X, Li L, et al. Concurrent chemoradiotherapy followed by adjuvant chemotherapy compared with concurrent chemoradiotherapy alone for the treatment of locally advanced nasopharyngeal carcinoma: a retrospective controlled study. Curr Oncol. 2014;21(3):e408-e417. doi:10.3747/co.21.1777

13. Kassambara A, Kosinski M, Biecek P, Fabian S Package"survminer". Available from: https://cran.rstudio.com/web/packages/survminer/ survminer.pdf. Accessed August 5, 2018.

14. D'Agostino RB Jr. Propensity score methods for bias reduction in the comparison of a treatment to a non-randomized control group. Stat Med. 1998;17(19):2265-2281. doi:10.1002/(ISSN)1097-0258
15. Susanne S. Propensity score based data analysis. Available from: https://cran.r-project.org/web/packages/nonrandom/vignettes/nonran dom.pdf. Accessed March 28, 2014.

16. Yang XL, Wang Y, Liang SB, et al. Comparison of the seventh and eighth editions of the UICC/AJCC staging system for nasopharyngeal carcinoma: analysis of 1317 patients treated with intensity-modulated radiotherapy at two centers. BMC Cancer. 2018;18(1):606. doi:10.1186/s12885-018-4419-1

17. Pan JJ, Ng WT, Zong JF, et al. Proposal for the 8th edition of the AJCC/UICC staging system for nasopharyngeal cancer in the era of intensity-modulated radiotherapy. Cancer. 2016;122(4):546-558. doi:10.1002/cner.v122.4

18. Zong J, Lin S, Lin J, et al. Impact of intensity-modulated radiotherapy on nasopharyngeal carcinoma: validation of the 7th edition AJCC staging system. Oral Oncol. 2015;51(3):254-259. doi:10.1016/ j.oraloncology.2014.10.012

19. Huang WY, Lin CL, Lin CY, et al. Survival outcome of patients with nasopharyngeal carcinoma: a nationwide analysis of 13407 patients in Taiwan. Clin Otolaryngol. 2015;40(4):327-334. doi:10.1111/coa.12371

20. Xu C, Chen YP, Liu X, et al. Establishing and applying nomograms based on the 8th edition of the UICC/AJCC staging system to select patients with nasopharyngeal carcinoma who benefit from induction chemotherapy plus concurrent chemoradiotherapy. Oral Oncol. 2017;69:99-107. doi:10.1016/j.oraloncology.2017.04.015

21. Liu YC, Wang WY, Twu CW, et al. Prognostic impact of adjuvant chemotherapy in high-risk nasopharyngeal carcinoma patients. Oral Oncol. 2017;64:15-21. doi:10.1016/j. oraloncology.2016.11.008

22. Twu CW, Wang WY, Chen CC, et al. Metronomic adjuvant chemotherapy improves treatment outcome in nasopharyngeal carcinoma patients with postradiation persistently detectable plasma Epstein-Barr virus deoxyribonucleic acid. Int $J$ Radiat Oncol Biol Phys. 2014;89(1):21-29. doi:10.1016/j.jirobp.2014.01.052

23. Skillington SA, Kallogjeri D, Lewis JS Jr., Piccirillo JF. The role of adjuvant chemotherapy in surgically managed, p16-positive oropharyngeal squamous cell carcinoma. JAMA Otolaryngol Head Neck Surg. 2017;143(3):253-259. doi:10.1001/ jamaoto.2016.3353

24. Dong YY, Xiang C, Lu JX, et al. Concurrent chemoradiotherapy plus adjuvant chemotherapy versus concurrent chemoradiotherapy in locoregionally advanced nasopharyngeal carcinoma: a matched-pair multicenter analysis of outcomes. Strahlenther Onkol. 2016;192 (6):394-402. doi:10.1007/s00066-016-0970-3

25. Chen L, Hu CS, Chen XZ, et al. Concurrent chemoradiotherapy plus adjuvant chemotherapy versus concurrent chemoradiotherapy alone in patients with locoregionally advanced nasopharyngeal carcinoma: a phase 3 multicentre randomised controlled trial. Lancet Oncol. 2012;13(2):163-171. doi:10.1016/S1470-2045(11)70320-5

26. Peng H, Chen BB, Tang LL, et al. Prognostic value of nutritional risk screening 2002 scale in nasopharyngeal carcinoma: a large-scale cohort study. Cancer Sci. 2018;109(6):1909-1919. doi:10.1111/ cas. 13603

27. Miao J, Xiao W, Wang L, et al. The value of the Prognostic Nutritional Index (PNI) in predicting outcomes and guiding the treatment strategy of nasopharyngeal carcinoma (NPC) patients receiving intensity-modulated radiotherapy (IMRT) with or without chemotherapy. J Cancer Res Clin Oncol. 2017;143(7):1263-1273. doi:10.1007/s00432-017-2360-3

28. Zhang W, Chen Y, Chen L, et al. Importance of maintaining body weight for prevention of distant metastasis of nasopharyngeal carcinoma: an alternative workflow for cancer-risk assessment. $J$ Cancer. 2017;8(12):2269-2276. doi:10.7150/jca.19611

29. Wang Q, Li P, Wu W. A systematic analysis of immune genes and overall survival in cancer patients. BMC Cancer. 2019;19(1):1225. doi:10.1186/s12885-019-6414-6 
30. Badr M, Johrens K, Allgauer M, et al. Morphomolecular analysis of the immune tumor microenvironment in human head and neck cancer. Cancer Immunol Immunother. 2019;68(9):1443-1454. doi:10.1007/s00262-019-02378-w
31. Zhang L, Tang LQ, Chen QY, et al. Plasma Epstein-Barr viral DNA complements TNM classification of nasopharyngeal carcinoma in the era of intensity-modulated radiotherapy. Oncotarget. 2016;7 (5):6221-6230. doi:10.18632/oncotarget.6754

\section{Publish your work in this journal}

Cancer Management and Research is an international, peer-reviewed open access journal focusing on cancer research and the optimal use of preventative and integrated treatment interventions to achieve improved outcomes, enhanced survival and quality of life for the cancer patient.
The manuscript management system is completely online and includes a very quick and fair peer-review system, which is all easy to use. Visit http://www.dovepress.com/testimonials.php to read real quotes from published authors. 